\title{
OUVIDORIAS E CONFLITOS NO AMBIENTE DE TRABALHO: CAMINHOS PARA PESQUISA E PRÁTICA
}

Paulo Marzionna ${ }^{1}$

\begin{abstract}
Resumo
Este artigo visa destacar o papel central que as Ouvidorias podem exercer na gestão de conflitos no ambiente de trabalho no Brasil, indicando caminhos para o futuro da pesquisa acadêmica e o crescimento das Ouvidorias em organizações brasileiras. Conflitos são parte integrante de qualquer relação de trabalho, gerando impactos negativos e positivos em indivíduos, equipes e organizações. A centralidade deste tema leva organizações a buscarem gerir conflitos no ambiente de trabalho, de modo a minimizar suas consequências negativas sem impossibilitar a obtenção de seus benefícios. Nos EUA, em face das limitações do sistema judicial de resolução de conflitos, organizações têm adotado cada vez mais métodos alternativos de resolução de conflitos, especialmente arbitragem e mediação. Os dados atualmente disponíveis sobre o Brasil sugerem que as Ouvidorias podem estar, de fato, exercendo esse papel central de gestão de conflitos no ambiente de trabalho. No entanto, é necessário aprofundar a pesquisa sobre o tema, para compreender efetivamente como as Ouvidorias têm atuado no país diante de conflitos no ambiente de trabalho de diferentes naturezas. Ademais, destaca-se como as Ouvidorias podem exercer um papel central na gestão de temas como assédio moral dentro de organizações brasileiras. Por fim, indica-se a Ouvidoria brasileira como potencial líder na expansão de outros métodos alternativos de resolução de conflitos no ambiente de trabalho, como a mediação, e na estruturação de sistemas integrados de gestão de conflitos.
\end{abstract}

Palavras-chave: Conflitos Trabalhistas. Resolução Alternativa de Conflitos. Ouvidoria Interna. Mediação. Sistemas Integrados de Gestão de Conflitos.

1 Advogado, consultor e pesquisador. Mestre e Doutorando em Relações do Trabalho pela School of Industrial and Labor Relations, Cornell University, EUA. Graduado em Direito pela Universidade de São Paulo. Pós-Graduado em Administração de Empresas pela FGV-EAESP. (pef35@cornell.edu) 


\begin{abstract}
This paper highlights the central role that Ombudsman's Offices might play in the management of workplace conflicts in Brazil, suggesting paths for future academic research and for the growth of Ombudsman's Offices in Brazilian organizations. Conflicts are an integral part of any employment relationship, generating negative and positive impacts in individual workers, teams and organizations. The centrality of this topic leads organizations to attempt to manage workplace conflicts, in order to minimize their negative consequences without losing their potential benefits. In the USA, due to the limitations of the litigation system, organizations have increasingly adopted alternative dispute resolution methods, especially employment arbitration and employment mediation. The data currently available on Brazil suggest that Ombudsman's Offices may, in fact, be playing the central role of conflict management in the workplace in the country. However, it is necessary to deepen the research on the topic, in order to effectively understand how Ombudsman's Offices in Brazil have responded to different types of workplace conflicts. Furthermore, it is emphasized how Ombudsman's Offices can play a central role in the management of issues like workplace bullying in Brazilian organizations. Finally, Ombudsmen in Brazil are identified as potential leaders in the expansion of alternative methods of conflict resolution in the workplace, such as mediation, and in the structuring of integrated conflict management systems.
\end{abstract}

Keywords: Workplace Conflicts. Alternative Dispute Resolution. Internal Organizational Ombudsman. Employment Mediation. Integrated Conflict Management Systems. 


\section{INTRODUÇÃO}

Conflitos são parte integrante das relações de trabalho e da vida organizacional, com diversas consequências no plano individual, de equipe e organizacional. Neste sentido, é natural que a sociedade e as organizações busquem administrar os conflitos de modo a dirimir as consequências negativas e potencializar os eventuais benefícios. No entanto, a limitação inerente aos métodos tradicionais de resolução de conflitos por via judicial levou à expansão acelerada de métodos alternativos, inclusive com relação ao ambiente de trabalho.

Este artigo busca analisar os dados disponíveis sobre a adoção de métodos alternativos de resolução de conflitos nos EUA e compará-los com os dados disponíveis sobre organizações brasileiras, com especial destaque para o papel exercido pelas Ouvidorias no ambiente de trabalho. Conclui-se com considerações sobre os futuros caminhos a serem percorridos pela pesquisa acadêmica sobre as Ouvidorias no ambiente de trabalho brasileiro, bem como os caminhos para a expansão das Ouvidorias em organizações brasileiras.

Frise-se que a opção por utilizar os EUA como referencial é motivada não só pela grande disponibilidade de informações de qualidade sobre a utilização de métodos alternativos de resolução de conflitos por organizações naquele país, mas porque é inegável que, no mundo organizacional e da administração, as práticas adotadas nos EUA são muitas vezes observadas e reproduzidas por organizações em diversos países, muitas vezes tomadas como sinônimo de um discutível conceito de "melhores práticas de gestão" (PUDELKO e HARZING, 2007). No entanto, não é raro que o contexto institucional americano seja ignorado quando da opção pela mera reprodução de prática de gestão em outro país. Desse modo, embora a opção por usar os EUA como referencial comparativo seja natural, neste artigo toma-se o cuidado de destacar peculiaridades institucionais que explicam as diferenças com relação aos fenômenos observados nos EUA e no Brasil.

\section{A IMPORTÂNCIA DOS CONFLITOS NO AMBIENTE DE TRABALHO}

O tema dos conflitos no ambiente de trabalho é de evidente importância, independentemente da visão teórica que se utilize para estudar as relações de trabalho. Embasamentos teóricos diferentes, no entanto, levarão a diferentes interpretações quanto às origens e consequências desses conflitos. Por exemplo, para a disciplina de Relações do Trabalho, conflitos são presentes e recorrentes em qualquer relação de trabalho, em razão das disparidades de poder que caracterizam essa relação. Para a disciplina de Recursos Humanos, entretanto, conflitos não são consequência de disparidade de forças, mas resultam de problemas de gestão. As diferentes compreensões do mesmo fenômeno também impactam as soluções apresentadas e defendidas em cada campo do conhecimento - para as Relações do Trabalho a solução passa pela relação sindicato-empresa, enquanto para a área de Recursos Humanos a solução reside em melhoria nas ferramentas de gestão (LEWIN, 2001).

O estudo desses conflitos é relevante não só pela massiva presença do fenômeno nas relações de trabalho, mas principalmente pelas consequências que o conflito pode trazer para trabalhadores 
e organizações. O tema já foi objeto de estudo de diversas disciplinas. Nas Relações do Trabalho, a presença de conflitos no ambiente de trabalho foi identificada como correlacionada negativamente com produtividade, eficiência e qualidade em plantas industriais (KATZ et al., 1985), seja pelo tempo despendido por gestores e trabalhadores na resolução de conflitos, seja pela resposta negativa de trabalhadores ao que for interpretado como tratamento injusto no ambiente de trabalho (ICHNIOWSKI, 1986).

Os impactos dos conflitos no ambiente de trabalho, no entanto, vão além da produtividade ou eficiência no nível empresarial, afetando diretamente indivíduos e equipes de trabalhadores, como demonstrado recorrentemente por estudos de psicologia e comportamento organizacional desde a década de 1990. Estudos nessa área demonstraram que a questão do conflito no ambiente de trabalho é complexa. Por um lado, conflitos de relacionamento de natureza pessoal costumam trazer impactos negativos para o desempenho de grupos no ambiente de trabalho. Por outro, há conflitos sobre a natureza das tarefas a serem conduzidas no ambiente de trabalho e a forma de conduzi-las, os quais, em determinadas situações e volumes, são positivos para a produtividade, eficiência e qualidade do trabalho da equipe (JEHN, 1995), e muitas vezes ligados ao aumento da capacidade de inovação de determinadas equipes (DE DREU, 2006).

A compreensão de que determinados tipos de conflitos podem gerar resultados positivos tem um importante impacto na vida de qualquer organização: idealmente, não basta suprimir a existência de qualquer conflito organizacional ou extirpar suas origens, sob o risco de perder também os benefícios que determinados conflitos podem trazer (RAHIM, 2002). Em realidade, as organizações devem buscar mecanismos para a gestão dos conflitos, numa tentativa de obter os benefícios que podem deles advir, constituindo aquilo que TJOSVOLD (2008) chama de organizações com conflitos positivos (conflict positive organizations).

Neste momento, resta evidente ser necessário que o leitor tenha uma conceituação mais ampla do que significa o conflito no ambiente de trabalho do que aquela usualmente utilizada no dia a dia. Por exemplo, é claro que discordâncias sobre pagamento de horas extras constitui conflito no ambiente de trabalho. Igualmente, também são conflitos as desavenças pessoais entre empregados, ou entre empregados e gestores, que em casos extremos podem chegar a configurar assédio moral ou outro tipo de falta grave de comportamento. Mas, da mesma forma, são conflitos as discordâncias entre equipes sobre quem é responsável por conduzir determinada atividade na organização, ou sobre a melhor forma de alcançar determinado objetivo ou corrigir certo problema. Com o entendimento dos conflitos no ambiente de trabalho como fenômeno complexo e multifacetado, fica fácil identificar inúmeras mudanças no mundo do trabalho que levam ao aumento das ocorrências de conflito trabalhista em suas múltiplas formas, tais como mudanças na organização do trabalho em razão de fatores externos, como globalização e tecnologia, ou fatores internos, como novas formas de estruturas organizacionais (LIPSKY et al., 2016).

Em face dos conceitos apresentados até o momento, resta evidente o papel central reservado ao tema da gestão dos conflitos no ambiente de trabalho no mundo atual, razão pela qual as próximas páginas são dedicadas à compreensão dos diferentes mecanismos de resolução de conflitos adotados por diferentes organizações, com especial atenção ao papel reservado às Ouvidorias nas organizações brasileiras. 


\section{MÉTODOS ALTERNATIVOS DE RESOLUÇÃO DE CONFLITOS}

$\mathrm{Na}$ última seção, discutiu-se o conceito de conflitos no ambiente de trabalho, seus impactos e sua importância. Nesta seção são analisados os diferentes mecanismos disponíveis para as organizações lidarem com os referidos conflitos.

O mecanismo mais tradicional para a resolução de qualquer tipo de conflito é a via judicial - o que não significa que seja o caminho mais adequado para isso. Afora os custos e o tempo despendido na via judicial, as soluções possíveis de serem alcançadas por essa via geralmente encontram-se limitadas a reparações financeiras, salvo algumas exceções (com resultados de obrigações de fazer ou não fazer). No campo dos conflitos de natureza trabalhista, tais características muitas vezes representam um mecanismo inadequado para lidar com os diversos tipos de conflitos que marcam a relação de trabalho, como descrito anteriormente.

Em face das limitações da via judicial como mecanismo de resolução de conflitos, as últimas décadas viram em todo o mundo o avanço de diversos métodos alternativos nesse campo. Frise-se que tal movimento é observável para os mais diversos tipos de conflitos, não só os de natureza trabalhista. Mecanismos como mediação, arbitragem, med-arb, Ouvidorias, painéis de pares (peer review panels), políticas de portas abertas e outros ganharam espaço nos mais variados setores e países (ver, por exemplo, BENSON, 2012; PURCELL, 2010; ROCHE e TEAGUE, 2011).

\subsection{O Caso dos EUA}

Em 1997, pesquisa realizada com as maiores empresas americanas de diversos setores (Fortune 1000 companies) revelou o quão presentes na vida organizacional americana eram os mecanismos alternativos de resolução de conflitos. Naquele momento, o percentual de empresas respondentes que afirmaram ter tido experiências nos três anos anteriores com mediação e arbitragem foi de $88 \%$ e $80 \%$, respectivamente. Apenas $10 \%$ das empresas respondentes afirmaram ter experiência com o uso de Ouvidorias (Ombudsman)2. Dentre os respondentes, 18,7\% afirmaram usar mediação frequentemente ou muito frequentemente, e 20,6\% afirmaram usar arbitragem frequentemente ou muito frequentemente (LIPSKY e SEEBER, 1998).

Em 2011 essa pesquisa foi conduzida novamente nos EUA, apontando algumas mudanças na utilização de mecanismos alternativos de resolução de conflitos nesse intervalo de catorze anos. O índice de utilização de mediação subiu de $87 \%$ para $98 \%$ e o índice de utilização de arbitragem subiu de $80 \%$ para $83 \%$. O número de empresas com experiência com Ouvidoria, por sua vez, subiu de $10 \%$ para $14 \%$ (STIPANOWICH e LAMARE, 2014).

Especificamente com relação a conflitos de natureza trabalhista, o uso de mediação subiu de $78,6 \%$ em 1997 para $85,5 \%$ em 2011. No entanto, o uso de arbitragem para casos trabalhistas observou uma queda de 62,2\% em 1997 para 37,8\% em 2011, sugerindo um possível desgaste no

2 Optou-se por omitir aqui outros métodos cobertos pela referida pesquisa, em face do objetivo do presente artigo. 
uso de arbitragem para conflitos desta natureza ${ }^{3}$. Atualmente, $87,4 \%$ das empresas respondentes afirmam ser provável ou muito provável que utilizem mediação para conflitos trabalhistas no futuro (em 1997 o índice era 84\%), comparado com apenas 38,9\% de empresas respondentes que afirmam ser provável ou muito provável que utilizem arbitragem para conflitos trabalhistas no futuro (em 1997 o índice era 71\%) (STIPANOWICH e LAMARE, 2014).

As pesquisas mencionadas acima revelaram mais do que a proporção da adoção de métodos individuais de resolução de conflitos por empresas americanas. Na realidade, desde 1997 os resultados apontam na direção de uma popularização na implementação de sistemas integrados de gestão de conflitos no ambiente de trabalho - com presença estimada em $20 \%$ das maiores organizações americanas em 1997 (LIPSKY e AVGAR, 2010). Em 2011, a estimativa é de que tais sistemas estejam presentes em ao menos $35 \%$ das maiores empresas americanas (STIPANOWICH e LAMARE, 2014).

Há vasta literatura disponível sobre as características principais de um sistema integrado de gestão de conflitos (para um resumo, ver ROCHE e TEAGUE, 2014). Via de regra, um sistema de gestão de conflitos deve: 1) ser abrangente (disponível para todos na organização e para todos os tipos de conflitos); 2) ter uma cultura de tolerância e resolução em estágios iniciais (deve tolerar discordâncias e incentivar a resolução nos estágios iniciais dos conflitos); 3) oferecer múltiplos canais de acesso; 4) oferecer múltiplos métodos de resolução de conflitos; e 5) possuir estrutura de apoio adequada, que coordene os múltiplos canais de acesso e métodos disponíveis e que traga a questão da gestão de conflitos para o dia a dia da organização (LIPSKY e AVGAR, 2010).

\subsection{O Caso Brasileiro}

No Brasil inexiste estudo com a extensão e profundidade dos acima citados. No entanto, há alguns indicativos importantes sobre o crescimento da utilização de mecanismos alternativos de resolução de conflitos ao longo das últimas décadas. Tendo como foco disputas de natureza comercial, pesquisa conduzida por LEMES (2017) em seis das principais câmaras de arbitragem brasileiras revela o aumento de 95\% no número total de casos levados à arbitragem por ano, entre 2010 e 2016. No mesmo sentido, a Câmara de Comércio Internacional aponta o Brasil como o quinto país no ranking da própria câmara em relação ao número total de arbitragens no ano de 2016 (VIVIANI, 2016). Os números sobre mediação extrajudicial também são limitados, embora a promulgação da Lei Brasileira de Mediação (Lei 13.140/2015) seja reveladora quanto à importância que o tema tem recebido em conflitos das mais diversas naturezas.

No caso específico dos conflitos de natureza trabalhista, por muito tempo os tribunais colocaram barreiras quanto à utilização de métodos alternativos que estivessem fora do domínio do judiciário. Os casos mais evidentes dizem respeito às impossibilidades quanto à arbitragem trabalhista, comumente entendida como inaplicável a conflitos individuais em face da indisponibilidade dos

3 Tal queda na utilização da arbitragem possivelmente está ligada à perda de suas características como método simples, rápido e barato, aproximando-se cada vez mais do processo judicial que visava substituir.

90 | Revista Científica da Associação Brasileira de Ouvidores/Ombudsman - Ano 2 - nº $2-2019$ 
direitos do trabalhador. De igual modo, o enfraquecimento das Comissões de Conciliação Prévia no início dos anos 2000 também pode ser entendido como sinal da resistência do judiciário trabalhista quanto à solução de conflitos fora dos tribunais. Isso, no entanto, não significa que o judiciário trabalhista se encontrasse isento de críticas, ou completamente fechado às alternativas ao tradicional processo judicial. Os Núcleos Permanentes de Conciliação, presentes em todos os tribunais trabalhistas, bem como as resoluções do Conselho Superior da Justiça do Trabalho (Res. 174/2016) reforçam o papel central que métodos alternativos de resolução de conflitos têm dentro do próprio judiciário trabalhista. Por fim, a nova redação do art. 507-A da Consolidação das Leis do Trabalho, trazida pela Reforma Trabalhista de 2017, passa a autorizar expressamente a assinatura de cláusula arbitral em contratos individuais de trabalho para empregados com remuneração no mínimo duas vezes superior ao limite máximo estabelecido para os benefícios do Regime Geral de Previdência Social. Tal alteração no texto da CLT reforça ainda mais o papel central que os métodos alternativos de solução de conflitos podem ocupar nas relações de trabalho no Brasil.

Embora o parágrafo anterior detalhe os limites à adoção formal de métodos alternativos de resolução de conflitos na seara trabalhista, é evidente que os conflitos nas relações de trabalho seguiram relevantes no país. O judiciário trabalhista, no entanto, não se apresentou como alternativa capaz de dirimir todos eles em função de limitações próprias do mecanismo da via judicial, o que sugere que se olhe para os diferentes métodos, ações e estratégias adotados dentro das empresas e organizações brasileiras para lidar com tais conflitos.

Nesse sentido, há alguns indicativos de que as Ouvidorias podem exercer um papel central no ambiente organizacional brasileiro no que diz respeito aos conflitos no ambiente de trabalho. Em matéria publicada na revista Você S/A sobre canais utilizados por empresas para lidar com o tema assédio moral, há a informação de que Ouvidorias estão presentes em 112 das 150 empresas que faziam parte do ranking de 2010 das melhores empresas para se trabalhar (INOHARA, 2013). Em 2016, pesquisa patrocinada pela Associação Brasileira de Recursos Humanos - ABRH também identificou algumas informações relevantes quanto aos métodos utilizados para gestão de conflitos no ambiente de trabalho. A pesquisa, respondida por representantes de 136 empresas, apontou que a mediação gerencial, adotada por pouco mais de $75 \%$ das organizações, é o mecanismo mais popular de gestão interna de conflitos, seguida pela mediação com recursos internos, adotada por pouco mais de $60 \%$ das organizações. Os "canais internos para colaboradores, Ouvidoria interna ou Ombudsman" foram apontados como utilizados por quase 30\% dos respondentes (LEVY et al., 2016).

Evidentemente, os números acima descritos devem ser olhados com certo cuidado, já que falta clareza na definição do que seja considerado mediação e, principalmente, Ouvidoria. Há um claro risco de se estar tratando meros canais internos de denúncias ou de reclamações como se equivalessem à Ouvidoria, ignorando as características essenciais desse instituto, como neutralidade, imparcialidade, independência e confidencialidade (ROWE e GADLIN, 2014). De qualquer maneira, tais dados apontam na direção de uma presença das Ouvidorias como canal para a gestão de conflitos internos significativamente mais relevante no Brasil do que em comparação com os dados atualmente disponíveis sobre os EUA: os dois números brasileiros disponíveis (75\% e aproximadamente 30\%) são bastante superiores aos 14\% americanos identificados na pesquisa de 2011 analisada na seção anterior. 


\section{POSSÍVEIS CAMINHOS DA PESQUISA SOBRE OUVIDORIAS NO BRASIL}

As hipóteses para o papel central exercido pelas Ouvidorias no ambiente de trabalho brasileiro, conforme descrito na seção anterior, são variadas. Passam pela barreira institucional a outros mecanismos, como arbitragem e mediação extrajudicial, mas podem também refletir o histórico de sucesso da utilização das Ouvidorias brasileiras para lidar com outros tipos de conflitos, notadamente envolvendo consumidores e usuários de serviços públicos. Se nos EUA empresas sem sindicato olharam para o ambiente sindical para buscar inspiração (COLVIN, 2003), é possível se argumentar que no Brasil organizações olharam para suas experiências com outros tipos de conflitos para buscar inspiração sobre como tratar os conflitos de natureza trabalhista. Tal fenômeno é identificado pela sociologia como isomorfismo institucional (DIMAGGIO e POWELL, 1983).

Entender as origens e os fatores que colocam as Ouvidorias em lugar de destaque no ambiente de trabalho brasileiro tem valor que vai além da mera curiosidade acadêmica. Responder a estas perguntas pode ajudar pesquisadores e profissionais de Ouvidoria a identificar o papel que as Ouvidorias podem de fato exercer nas organizações brasileiras com relação ao conflito no ambiente de trabalho e como elas se encaixam no sistema geral de resolução de conflitos composto por ferramentas internas e externas à organização, como o Poder Judiciário.

Como destacado anteriormente, os dados hoje disponibilizados pelas pesquisas brasileiras já citadas sofrem com a falta de uma definição clara sobre o que é a Ouvidoria no contexto do ambiente de trabalho organizacional. É evidente que uma maior clareza sobre o conceito da Ouvidoria no ambiente de trabalho é essencial para a correta mensuração de sua relevância nas relações de trabalho brasileiras, mas a missão pode ser mais complexa do que parece. Em pesquisa qualitativa realizada com empresas brasileiras de diversos setores, verificou-se que Ouvidorias voltadas para o público interno das organizações podem apresentar as mais diversas características quanto ao seu papel, métodos e estratégias adotadas diante dos mais variados temas. Por exemplo, identificou-se: 1) estratégias diversas diante de diferentes tipos de conflitos trabalhistas (ex.: assédio moral, assédio sexual, conflitos comportamentais etc.); 2) estratégias diversas diante dos sindicatos da categoria; e 3) diferentes posições da Ouvidoria dentro da estrutura organizacional e do organograma (MARZIONNA, 2016).

Desse modo, a pesquisa sobre o papel das Ouvidorias no ambiente de trabalho brasileiro hoje parece ter dois importantes campos a cobrir antes de tentar alçar voos mais altos quanto às perguntas que deve buscar responder. De um lado, a pesquisa sobre Ouvidorias deve se encaixar no esquema maior da ainda incipiente pesquisa sobre métodos alternativos de resolução de conflitos no ambiente de trabalho brasileiro, de modo a identificar os métodos mais utilizados pelas organizações no Brasil e as razões por trás dessas escolhas. Por outro lado, há espaço para a pesquisa acadêmica identificar de fato qual o papel hoje exercido pelas Ouvidorias nos conflitos no ambiente de trabalho brasileiro: quem é o Ouvidor? Onde a Ouvidoria se encaixa na estrutura da organização? Quais os métodos e estratégias adotadas pela Ouvidoria? Qual o grau de independência que a Ouvidoria brasileira possui para lidar com os conflitos de natureza trabalhista? Estas e tantas outras perguntas devem ser respondidas de modo a se traçar um retrato cada vez mais claro das Ouvidorias no país. 
As primeiras perguntas sobre o panorama geral da utilização de métodos alternativos de resolução de conflitos no ambiente de trabalho brasileiro ainda dependem de um grande projeto nos moldes do conduzido nos EUA com as mil maiores empresas do país. Hoje, no entanto, já se está mais próximo de responder ao segundo grupo de perguntas quanto ao papel das Ouvidorias no ambiente de trabalho brasileiro. No primeiro semestre de 2018, em conjunto com a Associação Brasileira de Ouvidores, o autor conduziu a primeira pesquisa quantitativa sobre o tema no Brasil. O questionário, respondido por 275 profissionais de Ouvidoria em todo o país, cobre temas como os tipos de demandas trabalhistas que chegam às Ouvidorias, as técnicas e estratégias adotadas pelos Ouvidores com relação aos conflitos de natureza trabalhista, a relação existente com sindicatos da categoria, entre outros tópicos. Os resultados definitivos da pesquisa serão objeto de outro artigo no futuro, mas apontam no sentido de forte atuação das Ouvidorias em organizações brasileiras com relação a conflitos de natureza trabalhista, além de indicar um alto nível de utilização de mediação, por parte dos Ouvidores, como método para resolução desses conflitos.

\section{POSSÍVEIS CAMINHOS DA OUVIDORIA NO AMBIENTE DE TRABALHO BRASILEIRO}

Se os caminhos para a pesquisa sobre Ouvidorias no ambiente de trabalho brasileiro estão abertos e são promissores, o mesmo pode se afirmar sobre as possibilidades que se abrem na prática para Ouvidorias em organizações brasileiras. Embora não se negue a importância do tema dos conflitos no ambiente de trabalho e se reconheçam as limitações do judiciário para lidar adequadamente com os diversos tipos de conflito dessa natureza, o ambiente institucional brasileiro apresentou ao longo das últimas décadas uma série de barreiras para o desenvolvimento dos mecanismos alternativos mais popularmente adotados em países como os EUA. Nessas circunstâncias, observou-se o florescimento das Ouvidorias como importante canal para lidar com esses conflitos, muito mais presentes no Brasil do que no contexto norte-americano. Nessa conjuntura, quais os possíveis caminhos que se abrem para as Ouvidorias no país no que diz respeito ao ambiente de trabalho organizacional?

Há razões para acreditar que o futuro pode elevar as Ouvidorias a uma posição ainda mais destacada no ambiente de trabalho brasileiro. Em um sistema de relações do trabalho em que o tema de assédio moral ocupa um papel cada vez mais central na vida organizacional, as Ouvidorias possuem características próprias, como independência e imparcialidade, que as tornam preparadas para lidar com tais temas (MARCELINO, 2017). Tais características faltam a outros departamentos, como Recursos Humanos, por exemplo, muitas vezes vistos como próximos demais da gestão, notadamente em modelos de consultoria interna (HARRINGTON et al., 2012).

Ademais, é possível enxergar nas Ouvidorias o potencial de liderar a expansão de métodos alternativos de resolução de conflitos no ambiente de trabalho, potencialmente capitaneando a construção de sistemas integrados de gestão de conflitos. É da natureza da Ouvidoria a adoção de diferentes métodos de resolução de conflitos, bem como a disponibilidade de diferentes canais de acesso àqueles por ela atendidos. Resultados preliminares da citada pesquisa com profissionais de Ouvidoria apontaram no sentido de ampla utilização de técnicas de mediação e crença no espaço para que a mediação seja ainda mais utilizada no futuro. 
Por fim, a autorização da utilização da arbitragem trabalhista pela Reforma Trabalhista de 2017 é um sinal claro no sentido de maior abertura institucional para a adoção de diferentes métodos de resolução de conflitos. Nesse contexto, a Ouvidoria, já inserida no ambiente organizacional brasileiro, pode se utilizar de seu conhecimento prévio sobre a realidade organizacional para capitanear a expansão dos métodos alternativos de resolução de conflitos no ambiente de trabalho.

\section{REFERÊNCIAS}

BENSON, J. Alternative Dispute Resolution in Japan: The Rise of Individualism. The International Journal of Human Resource Management, 23(3), 511-527, 2012.

COLVIN, A. J. Institutional Pressures, Human Resource Strategies, and the Rise of Nonunion Dispute Resolution Procedures. Industrial and Labor Relations Review, 56(3), 375-392, 2003.

DE DREU, C. K. When Too Little or Too Much Hurts: Evidence for a Curvilinear Relationship Between Task Conflict and Innovation in Teams. Journal of Management, 32(1), 83-107, 2006.

DIMAGGIO, P. J.; POWELL, W. W. The Iron Cage Revisited: Institutional Isomorphism and Collective Rationality in Organizational Fields. American Sociological Review, 48(2), 147-160, 1983.

HARRINGTON, S.; RAYNER, C.; WARREN, S. Too Hot to Handle? Trust and Human Resource Practitioners' Implementation of Anti-Bullying Policy. Human Resource Management Journal, 22(4), 392-408, 2012.

ICHNIOWSKI, C. The Effects of Grievance Activity on Productivity. Industrial and Labor Relations Review, 40(1), 7589, 1986.

INOHARA, A. O 0800 explode no combate ao assédio moral. Exame - Você S/A., 28 nov. 2013. Disponível em: <https://exame.abril.com.br/carreira/a-explosao-do-0800/2/>. Acesso em: 28 maio 2018.

JEHN, K. A. A Multimethod Examination of The Benefits and Detriments of Intragroup Conflict. Administrative Science Quarterly, 256-282, 1995.

KATZ, H. C.; KOCHAN, T. A.; WEBER, M. R. Assessing the Effects of Industrial Relations Systems and Efforts to Improve the Quality of Working Life on Organizational Effectiveness. Academy of Management Journal, 28(3), 509-526, 1985.

LEMES, S. F. Pesquisa: Arbitragem em Números e Valores. 2017. Disponível em: <http://selmalemes.adv.br/artigos/ An $\%$ C3\%A1lise- $\% 20$ Pesquisa-\%20Arbitragens $\% 20 N s \% 20 \% 20$ e $\% 20$ Valores $\% 20 \_2010 \% 20 a \% 202016$ _.pdf $>$. Acesso em: 28 maio 2018.

LEVY, F.; BURBRIDGE, M.; FREIRE, M.; SILVA, T. O Papel do RH na Gestão de Conflitos Organizacionais. ABRH-SP - Associação Brasileira de Recursos Humanos. 2016. Disponível em: <https://abrhsp.org.br/wp-content/uploads/2018/03/resultado_rh_resolucao_2016.pdf>. Acesso em: 28 maio 2018.

LEWIN, D. IR and HR Perspectives on Workplace Conflict: What Can Each Learn From the Other? Human Resource Management Review, 11(4), 453-485, 2001.

LIPSKY, D. B.; AVGAR, A. C. The Conflict Over Conflict Management. Dispute Resolution Journal, 65(2/3), $11,2010$.

LIPSKY, D. B.; AVGAR, A. C.; LAMARE, J. R. Introduction: New Research on Managing and Resolving Workplace Conflict: Setting the Stage. Advances in Industrial and Labor Relations, 22, ix - xxxi, 2016.

LIPSKY, D. B.; SEEBER, R. L. The Appropriate Resolution of Corporate Disputes: A Report on the Growing Use of ADR by US Corporations. Cornell/Perc Institute on Conflict Resolution, 1998.

MARCELINO, K. J. Possibilidades e desafios das Ouvidorias no enfrentamento de denúncias de assédio moral no ambiente de trabalho. Revista Científica da Associação Brasileira de Ouvidores/Ombudsman, 1(1), 83-91, 2017.

MARZIONNA, P. Complaining to the Ombudsman: alternative dispute resolution in the Brazilian workplace. 2016. Dissertação (Mestrado em Relações do Trabalho) - School of Industrial and Labor Relations, Cornell University, Ithaca, NY, EUA. 
PUDELKO, M.; HARZING, A. W. Country-of-Origin, Localization, or Dominance Effect? An Empirical Investigation of HRM Practices in Foreign Subsidiaries. Human Resource Management, 46(4), 535-559, 2007.

PURCELL, J. Individual Disputes at the Workplace: Alternative Disputes Resolution. Dublin, Irlanda: Eurofund, 2010.

RAHIM, M. A. Toward a Theory of Managing Organizational Conflict. International Journal of Conflict Management, 13(3), 206-235, 2002.

ROCHE, W. K.; TEAGUE, P. Firms and Innovative Conflict Management Systems in Ireland. British Journal of Industrial Relations, 49, 436-459, 2011.

Conflict Management Systems. In: ROCHE, W. K.; TEAGUE, P.; COLVIN, A. J. (Ed.). The Oxford handbook of conflict management in organizations. Oxford University Press, 2014.

ROWE, M.; GADLIN, H. The Organizational Ombudsman. In: ROCHE, W. K.; TEAGUE, P.; COLVIN, A. J. (Ed.). The Oxford handbook of conflict management in organizations. Oxford University Press, 2014.

STIPANOWICH, T. J.; LAMARE, J. R. Living with ADR: Evolving Perceptions and Use of Mediation, Arbitration, and Conflict Management in Fortune 1000 Corporations. Harvard Negotiation Law Review, 19, 1, 2014.

TJOSVOLD, D. The Conflict-Positive Organization: It Depends Upon Us. Journal of Organizational Behavior, 29(1), 19-28, 2008.

VIVIANI, L. Brasil é o $5^{\circ}$ país que mais utiliza arbitragem no mundo. Jota. 19 set. 2017. Disponível em: <https://www. jota.info/justica/brasil-e-o-5o-pais-que-mais-utiliza-arbitragem-no-mundo-19092017>. Acesso em: 28 maio 2018. 